\title{
Extended Hamiltonians, Coupling-Constant Metamorphosis and the Post-Winternitz System ${ }^{\star}$
}

\author{
Claudia Maria CHANU, Luca DEGIOVANNI and Giovanni RASTELLI \\ Dipartimento di Matematica, Università di Torino, Torino, via Carlo Alberto 10, Italy \\ E-mail: claudiamaria.chanu@unito.it,luca.degiovanni@gmail.com,giovanni.rastelli@unito.it
}

Received September 26, 2015, in final form November 16, 2015; Published online November 24, 2015

http://dx.doi.org/10.3842/SIGMA.2015.094

\begin{abstract}
The coupling-constant metamorphosis is applied to modified extended Hamiltonians and sufficient conditions are found in order that the transformed high-degree first integral of the transformed Hamiltonian is determined by the same algorithm which computes the corresponding first integral of the original extended Hamiltonian. As examples, we consider the Post-Winternitz system and the 2D caged anisotropic oscillator.
\end{abstract}

Key words: superintegrable systems; extended systems; coupling-constant metamorphosis

2010 Mathematics Subject Classification: 37J35; 70H33

This article is dedicated to Sergio Benenti, our mentor, colleague and friend.

\section{Introduction}

Classical and quantum Hamiltonian systems depending on a rational parameter $\lambda$ and admitting first integrals, or symmetry operators, of degree determined by $\lambda$ have been recently the object of research in integrable systems theory, with a particular interest on superintegrable and separable systems. In many examples the configuration manifolds of these systems are constantcurvature Riemannian or pseudo-Riemannian manifolds of finite dimension $[1,13,14,18]$, but some examples of non-constant-curvature manifolds are also known [11].

In a series of articles, we proposed an algorithm for the construction, given a suitable $N$ dimensional Hamiltonian $L$, of classical $(N+2)$-dimensional Hamiltonians with first integrals depending on a rational parameter; this approach started from the analysis of the JacobiCalogero and Wolfes systems [3]. The construction is not restricted to superintegrable or separable systems, even if it allows to build new superintegrable systems from known ones [5]. Although it involves a privileged coordinate system, the application of the algorithm is intrinsically characterized [4] and it is ultimately rooted into the geometry of the manifold (Poisson or symplectic) where $L$ is defined, imposing conditions, as instance, on Riemannian curvature, on the geometry of warped manifolds [8] (see also, for example, $[16,20]$ ) and on a particular type of master symmetries $[4,17]$.

The Hamiltonian systems admitting such an algorithmic construction are called "extensions" and many of the known Hamiltonians with high-degree first integrals depending on $\lambda$ are indeed extensions of some other Hamiltonian $L$ [7]. The algorithm consists essentially in the determination of the $\lambda$-dependent first integral through the power of a differential operator generated by the Hamiltonian vector field of $L$, applied to some suitable function $G$. We stress the fact that our construction of the $\lambda$-dependent first integral does not assume integrability, separability or superintegrability of the system, differently from all other approaches, and provides a compact expression of the real first integral itself.

\footnotetext{
${ }^{\star}$ This paper is a contribution to the Special Issue on Analytical Mechanics and Differential Geometry in honour of Sergio Benenti. The full collection is available at http://www.emis.de/journals/SIGMA/Benenti.html
} 
With this article we modify the algorithm in order to apply it to the Post-Winternitz (PW) system. The PW-system is a $\lambda$ dependent Hamiltonian system including a Kepler-Coulomb potential term [15] originally obtained by coupling-constant metamorphosis (CCM) [9] of the Tremblay-Turbiner-Winternitz (TTW) system [21]. The CCM is a powerful tool for obtaining new integrable or superintegrable Hamiltonian systems from known ones (in particular, when applied to Stäckel separable systems it is called "Stäckel transform" [2]) and has been recently extensively employed in the study and classification of superintegrable Hamiltonian systems (see $[12,14]$ and references therein).

It is proved in [7] that the TTW system is what we call a "modified extension" and, therefore, that its $\lambda$-dependent first integral can be computed through the power of some operator $W$ applied to a function $G$. In the following, we apply the CCM to modified extensions and show in particular that the $\lambda$-dependent first integral of the PW system is equal to some power of the CCM of the operator $W$ applied to the same function $G$ appearing in the construction of the TTW system as extension. Therefore, even if the PW Hamiltonian cannot be written as a modified extension in the same way of the TTW system, nevertheless the same algorithm for the determination of the first integral works as well. This suggests the definition of a class of Hamiltonian systems which includes all the systems we call "extended Hamiltonians" and those systems related to them as the PW is related to the TTW.

In Section 2 we recall the definition of CCM and its connection with the PW system. In Section 3 we review the theory of extended Hamiltonian systems. In Section 4, where the main results are exposed, we study the application of CCM to extended systems and the results are completed by two examples: the TTW system, from which the PW system is obtained, and the caged anisotropic oscillator. In Section 5 the results of the article are summarized, a new class of Hamiltonian systems is defined, to include extended Hamiltonians and related systems; a new direction of research is suggested.

\section{Coupling-constant metamorphosis}

The coupling-constant metamorphosis (CCM) [12] transforms integrable or superintegrable systems in new integrable or superintegrable ones, by mapping first integrals in first integrals. We take the following statement from Theorem 1 of [15] (where the CCM is called Stäckel transform), Theorem 1 of [12] and, ultimately, from [9]

Theorem 1. Let us consider a Hamiltonian $H=\hat{H}-\tilde{E} U$ in canonical coordinates $\left(q^{i}, p_{i}\right)$, where $\hat{H}\left(q^{i}, p_{i}\right)$ is independent of the arbitrary parameter $\tilde{E}$ and $U\left(q^{i}\right)$, with an integral of the motion $K$ (depending on $\tilde{E})$. If we define the $C C M$ of $H$ and $K$ as $\tilde{H}=U^{-1}(\hat{H}-E)$ and $\tilde{K}=\left.K\right|_{\tilde{E}=\tilde{H}}$ then $\tilde{K}$ is an integral of the motion for $\tilde{H}$.

For example, in [15] the superintegrability of the Post-Winternitz (PW) system of Hamiltonian

$$
H_{\mathrm{PW}}=p_{r}^{2}+\frac{1}{r^{2}}\left(p_{\phi}^{2}+\frac{1}{4} f_{2}\left(\frac{\phi}{2}\right)\right)-\frac{Q}{2 r}
$$

where

$$
f_{2}(x)=k^{2}\left(\frac{\alpha}{\cos ^{2}(k x)}+\frac{\beta}{\sin ^{2}(k x)}\right)
$$

is proved for $k \in \mathbb{Q}$ by writing it as result of the CCM applied to the Tremblay-TurbinerWinternitz (TTW) system $[7,21]$

$$
H_{\mathrm{TTW}}=p_{\rho}^{2}+\frac{1}{\rho^{2}}\left(p_{\theta}^{2}+f_{2}(\theta)\right)-\tilde{E} \rho^{2} .
$$


Indeed, by applying to it the CCM, the TTW system (2) becomes a Hamiltonian system of Hamiltonian

$$
\tilde{H}=\frac{1}{\rho^{2}}\left(p_{\rho}^{2}+\frac{1}{\rho^{2}}\left(p_{\theta}^{2}+f_{2}(\theta)\right)-E\right),
$$

which coincides with (1), through the coordinate change $\rho=\sqrt{2 r}, \phi=2 \theta$, and by setting $E=Q / 2$.

In this example a system on the Euclidean plane is mapped into another system on the same manifold. This is not always the case: by applying the CCM to the 2D caged oscillator [10, 22]

$$
H_{\mathrm{co}}=\frac{1}{2} p_{y}^{2}+\frac{1}{2} p_{x}^{2}+\omega^{2}\left(k^{2} x^{2}+y^{2}\right)+\frac{b}{x^{2}}+\frac{c}{y^{2}},
$$

with $\tilde{E}=-c$, we get

$$
\tilde{H}=y^{2}\left(\frac{1}{2} p_{y}^{2}+\frac{1}{2} p_{x}^{2}+\omega^{2}\left(k^{2} x^{2}+y^{2}\right)+\frac{b}{x^{2}}-E\right),
$$

which is a system on the Poincaré half-plane.

\section{Extensions}

In [7] we show that the TTW system can be written as a modified extension. We recall that a $(N+2)$-dimensional Hamiltonian $H$ is a modified extension of the $N$-dimensional Hamiltonian $L$ if

i) there exist canonical coordinates $\left(u, p_{u}\right)$ such that

$$
H=\frac{1}{2} p_{u}^{2}+f(u)+\left(\frac{m}{n}\right)^{2} \alpha(u) L, \quad m, n \in \mathbb{N} \backslash\{0\},
$$

and the Hamiltonian $L$ does not depend on $\left(u, p_{u}\right)$;

ii) for some constants $c$ and $L_{0}$ not both vanishing, the equation

$$
X_{L}^{2}(G)=-2\left(c L+L_{0}\right) G
$$

where $X_{L}$ is the Hamiltonian vector field of $L$, admits a solution $G$, such that $X_{L}(G) \neq 0$;

iii) the functions $\alpha$ and $f$ are those given in Table 1 .

Then, given the operator $W$ defined by

$$
W\left(G_{\nu}\right)=\left(p_{u}+\frac{\mu}{\nu^{2}} \gamma(u) X_{L}\right)^{2}\left(G_{\nu}\right)+\delta(u) G_{\nu}
$$

with $(\mu, \nu)=(m, n)$ if $m$ is even, $(\mu, \nu)=(2 m, 2 n)$ if $m$ is odd, $\gamma$ and $\delta$ defined as in Table 1 and $G_{\nu}$ being the $\nu$-th element of the recursion

$$
G_{1}=G, \quad G_{\nu+1}=X_{L}\left(G_{1}\right) G_{\nu}+\frac{1}{\nu} G_{1} X_{L}\left(G_{\nu}\right),
$$

we have that

$$
K=W^{\frac{\mu}{2}}\left(G_{\nu}\right)
$$

is a first integral of $H$. 
Table 1. Functions involved in the modified-extensions of $L$.

\begin{tabular}{|c|c|c|}
\hline$\alpha=-\gamma^{\prime}=$ & $c=0$ & $c \neq 0$ \\
\hline$f=\frac{m^{2}}{n^{2}} L_{0} \gamma^{2}+\frac{f_{0}}{\gamma^{2}}=$ & $A$ & $\frac{c}{S_{\kappa}^{2}(c u)}$ \\
$\gamma=$ & $-A u$ & $\frac{m^{2}}{n^{2}} L_{0} A^{2} u^{2}+\frac{f_{0}}{A^{2} u^{2}}$ \\
$\frac{m^{2}}{n^{2}} \frac{L_{0}}{T_{\kappa}^{2}(c u)}+f_{0} T_{\kappa}^{2}(c u)$ \\
$\delta=\frac{2 f_{0}}{\gamma^{2}}=$ & $\frac{2 f_{0}}{A^{2} u^{2}}$ & $\frac{1}{T_{\kappa}(c u)}$ \\
\hline
\end{tabular}

In Table $1, A$ and $\kappa$ are arbitrary constants and the functions $S_{\kappa}$ and $T_{\kappa}$ are the trigonometric tagged functions

$$
\begin{aligned}
& S_{\kappa}(x)=\left\{\begin{array}{ll}
\frac{\sin \sqrt{\kappa} x}{\sqrt{\kappa}}, & \kappa>0, \\
x, & \kappa=0, \\
\frac{\sinh \sqrt{|\kappa|} x}{\sqrt{|\kappa|}}, & \kappa<0,
\end{array} \quad C_{\kappa}(x)= \begin{cases}\cos \sqrt{\kappa} x, & \kappa>0, \\
1, & \kappa=0, \\
\cosh \sqrt{|\kappa|} x, & \kappa<0,\end{cases} \right. \\
& T_{\kappa}(x)=\frac{S_{\kappa}(x)}{C_{\kappa}(x)}
\end{aligned}
$$

(see [6] and [19] for a summary of their properties).

We remark that

- If (6) has a solution for $c \neq 0$, then we may assume without loss of generality $L_{0}=0$, because $L$ is determined up to additive constants.

- In the case of natural Hamiltonians $L$, the possibility of finding extensions of $L$ is strictly related to the geometry of the base manifold of $L$ [4]. An intrinsic characterization of extended Hamiltonians $H$ is given in [4].

- The extension $H$ of a Hamiltonian $L$ with $k$ functionally independent first integrals is a Hamiltonian with $k+2$ functionally independent first integrals [7]. Indeed, $H$ itself and $W^{\frac{\mu}{2}}\left(G_{\nu}\right)$ are functionally independent first integrals of $H$ together with $L$ and all its functionally independent first integrals. If $L$ is maximally superintegrable, then also $H$ is.

- The dynamical equations in $\left(u, p_{u}\right)$ are always separated from those in the variables $\left(q^{i}, p_{i}\right)$ of $L$, being $L$ a constant of motion for $H$.

\section{Coupling-constant metamorphosis of extended Hamiltonians}

By Theorem 1, it follows that the CCM can be applied to any modified extension (5) by setting $\tilde{E}=-f_{0}, U=1 / \gamma^{2}$. Therefore, in this case we have

$$
\begin{aligned}
\hat{H} & =\frac{1}{2} p_{u}^{2}-\left(\frac{m}{n}\right)^{2} \gamma^{\prime} L+\frac{m^{2}}{n^{2}} L_{0} \gamma^{2}, \\
W & =\left(p_{u}+\frac{\mu}{\nu^{2}} \gamma(u) X_{L}\right)^{2}-2 \frac{\tilde{E}}{\gamma^{2}} .
\end{aligned}
$$

Moreover, the function

$$
\tilde{H}=\gamma^{2}(\hat{H}-E)=\frac{\gamma^{2}}{2} p_{u}^{2}-\left(\frac{m}{n}\right)^{2} \gamma^{2} \gamma^{\prime}(u) L+\frac{m^{2}}{n^{2}} L_{0} \gamma^{4}-E \gamma^{2}
$$


is the transformed Hamiltonian and

$$
\tilde{K}=\left(W^{\frac{\mu}{2}}\left(G_{\nu}\right)\right)_{\mid \tilde{E}=\tilde{H}},
$$

the transformed first integral of $\tilde{H}$.

A natural question is if $\tilde{K}$ is again given by a power of some operator applied to some function. The main result of this paper is that the answer is positive: $\tilde{K}$ can be computed by applying $\mu / 2$ times the operator

$$
\tilde{W}=\left.(W)\right|_{\tilde{E}=\tilde{H}}=\left(p_{u}+\frac{\mu}{\nu^{2}} \gamma X_{L}\right)^{2}+2(E-\hat{H}),
$$

to the same function $G_{\nu}$ used for the determination of $K$. Indeed,

Proposition 1. The transformed first integral $\tilde{K}$ of $(10)$ is $\tilde{W}^{\frac{\mu}{2}}\left(G_{\nu}\right)$, where

$$
\tilde{W}=2\left(\frac{\mu}{\nu^{2}} \gamma p_{u} X_{L}-\frac{\mu^{2}}{\nu^{2}}\left(\left(c \gamma^{2}-\gamma^{\prime}\right) L+\gamma^{2} L_{0}\right)+E\right)
$$

and $G_{\nu}$ is computed via the recursion (7).

Proof. We consider the iterated application of $\tilde{W}$. Being $W$ and $\tilde{H}$ given by (9) and (10) respectively, we have $W(\tilde{H})=\tilde{H} W$ because $X_{L}(\tilde{H})=0$. Moreover, $\tilde{W} X_{L}=X_{L} \tilde{W}$, therefore

$$
\left.\left(W^{\frac{\mu}{2}}\right)\right|_{\tilde{E}=\tilde{H}}=\left(\left.W\right|_{\tilde{E}=\tilde{H}}\right)^{\frac{\mu}{2}}=\tilde{W}^{\frac{\mu}{2}} .
$$

Finally, we remark that $G_{\nu}$ does not depend on $\tilde{E}$, as well as $L$. The explicit form of $\tilde{W}$ follows by expanding (11), inserting (8) in it and by applying the formula [6]

$$
X_{L}^{2}\left(G_{\nu}\right)=-2 \nu^{2}\left(c L+L_{0}\right) G_{\nu} .
$$

Remark 1. Let us consider the CCM of a natural $2 N$-dimensional Hamiltonian with a generic potential $U\left(q^{1}, \ldots, q^{N}, u\right)$. If $G_{\nu}$ and $L$ are independent from $\tilde{E}$, then the condition for having (13) is, from the proof of Proposition 1,

$$
X_{L}(\tilde{H})=-\frac{1}{U^{2}}(\hat{H}-E) X_{L}(U)=0,
$$

that, by requiring its validity on the whole space, is equivalent to

$$
X_{L}(U)=0 .
$$

For $L\left(q^{i}, p_{i}\right)$ such that $\partial L / \partial p_{i} \neq 0, i=1, \ldots, N$, the condition of above is equivalent to $U(u)$ and we go back to the result of Proposition 1. Other situations are possible for different $L$. We do not consider here the reduction of the system to the submanifold $\hat{H}=E$, i.e., $\tilde{H}=0$, and its first integrals.

Remark 2. Up to a rescaling of $\tilde{u}=\tilde{u}(u)$ such that

$$
\frac{d \tilde{u}}{d u}=\frac{1}{\gamma(u)} \quad \text { and } \quad p_{\tilde{u}}=\gamma p_{u}
$$

the Hamiltonian (10) is of the form (5), and the operator $\tilde{W}$ defined by (12) becomes

$$
\tilde{W}=\frac{2 \mu}{\nu^{2}} p_{\tilde{u}} X_{L}+2 \frac{\mu^{2}}{\nu^{2}} \delta_{1}(\tilde{u}) L+\delta_{2}(\tilde{u})
$$

where the functions $\delta_{i}$ are given in Table 2. The general (i.e., independent of CCM) conditions allowing the existence of first integrals generated by such type of operator will be analysed elsewhere. 
Table 2. Functions $\delta_{1}$ and $\delta_{2}$.

\begin{tabular}{|c|c|c|c|}
\hline & $c \neq 0, \kappa \neq 0$, & $c \neq 0, \kappa=0$, & $c=0, L_{0} \neq 0$ \\
\hline$\delta_{1}$ & $\frac{c \kappa\left(1+C_{\kappa}(c u)^{2}\right)}{1-C_{\kappa}(c u)^{2}}=\frac{c \kappa}{\tanh (\kappa c \tilde{u})}$ & $\frac{2}{c u^{2}}=\frac{1}{\tilde{u}}$ & $A$ \\
$\delta_{2}$ & $2 E+\frac{L_{0} \mu^{2} \kappa}{\nu^{2}}\left(\frac{1}{\tanh (c \kappa \tilde{u})}-1\right)$ & $2 E+\frac{L_{0} \mu^{2} \kappa}{\nu^{2} c \tilde{u}}$ & $2 E+2 \frac{\mu^{2}}{\nu^{2}} \frac{L_{0} A^{2}}{e^{2 A \tilde{u}}}$ \\
\hline
\end{tabular}

\subsection{Example 1: the TTW system}

In [7] it is shown that the TTW system (2) is a modified extension. Indeed, the extension of the Hamiltonian

$$
L=\frac{1}{2} p_{\psi}^{2}+\frac{c_{1}+c_{2} \cos \psi}{\sin ^{2} \psi},
$$

(satisfying (6) for $c=1, L_{0}=0$ and $G=p_{\psi} \sin \psi$ ) for $\kappa=0$, that is for $\gamma=1 / u$, is

$$
H=\frac{1}{2} p_{u}^{2}+\frac{m^{2}}{n^{2} u^{2}}\left(\frac{1}{2} p_{\psi}^{2}+\frac{c_{1}+c_{2} \cos \psi}{\sin ^{2} \psi}\right)+f_{0} u^{2} .
$$

The rescaling $u=\rho, \psi=2 k \theta$, the change of parameters

$$
\frac{m}{n}=k, \quad c_{1}=\alpha+\beta, \quad c_{2}=\beta-\alpha,
$$

and the position $\tilde{E}=-2 f_{0}$, transform (15) into the Hamiltonian $H_{\mathrm{TTW}}$ of (2) multiplied by 2 .

The PW Hamiltonian (1), instead, is not a modified extension, because the Kepler-Coulomb term $\frac{Q}{2 r}$ cannot be included in the form of $f$ given in Table 1 .

By applying the CCM based on $\tilde{E}=-f_{0}$ as in Theorem 1 to the Hamiltonian (15), we get

$$
\tilde{H}=\frac{1}{2 u^{2}} p_{u}^{2}+\frac{m^{2}}{n^{2} u^{4}}\left(\frac{1}{2} p_{\psi}^{2}+\frac{c_{1}+c_{2} \cos \psi}{\sin ^{2} \psi}\right)-\frac{E}{u^{2}}
$$

and, by performing the rescaling $u^{2}=2 r$, we obtain

$$
\tilde{H}=\frac{1}{2} p_{r}^{2}+\frac{m^{2}}{4 n^{2} r^{2}}\left(\frac{1}{2} p_{\psi}^{2}+\frac{c_{1}+c_{2} \cos \psi}{\sin ^{2} \psi}\right)-\frac{E}{2 r},
$$

which is, after (16), and the rescaling $\psi=2 k \phi$ together with the change of parameters $Q=2 E$, one half of the Hamiltonian (1).

Then, the operator generating first integrals of (17) for any rational $m / n$ is

$$
\tilde{W}=2\left(\frac{\mu}{\nu^{2} u} p_{u} X_{L}-\frac{\mu^{2}}{\nu^{2} u^{2}} L+E\right),
$$

with $(\mu, \nu)=(m, n)$ for $m$ even, $(\mu, \nu)=(2 m, 2 n)$ for $m$ odd and where $X_{L}$ is the Hamiltonian vector field of (14). The function $G_{\nu}$ is recursively determined by

$$
G_{1}=(\sin \psi) p_{\psi}, \quad G_{\nu+1}=X_{L}\left(G_{1}\right) G_{\nu}+\frac{1}{\nu} G_{1} X_{L}\left(G_{\nu}\right) .
$$

By the rescaling $u^{2}=2 r$, we get the operator generating first integrals for (18):

$$
\tilde{W}=\frac{2 \mu}{\nu^{2}} p_{r} X_{L}-\frac{\mu^{2}}{\nu^{2} r} L+2 E .
$$




\subsection{Example 2: the caged anisotropic oscillator}

In order to write the Hamiltonian on the Poincaré half-plane (4) as the CCM of a modified extension, we need to express the caged oscillator Hamiltonian (3) as a modified extension. From Section 3, we know that the expression of a modified extension in a plane when $c=0$ is

$$
H_{m, n}=\frac{1}{2} p_{u}^{2}-\frac{m^{2}}{n^{2}} \gamma^{\prime} L+\frac{m^{2}}{n^{2}} L_{0} \gamma^{2}+\frac{f_{0}}{\gamma^{2}},
$$

where $\gamma=-A u$. From [7] we know that the most general one-dimensional natural Hamiltonian $L\left(p_{q}, q\right)$ admitting an extension for $c=0$, with $G=\left(a_{1} q+a_{2}\right) p_{q}$, is

$$
L=\frac{1}{2} p_{q}^{2}+\frac{L_{0}}{4 a_{1}^{2}}\left(a_{1} q+a_{2}\right)^{2}+\frac{c_{1}}{\left(a_{1} q+a_{2}\right)^{2}}+c_{2},
$$

being $A, a_{i}, c_{i}, L_{0}$ real constants. By extending the Hamiltonian $L$ into (19), we can write the CCM of $H_{m, n}$ according to (10), obtaining

$$
\tilde{H}_{m, n}=\gamma^{2}\left(\frac{1}{2} p_{u}^{2}-\frac{m^{2}}{n^{2}} \gamma^{\prime} L+\frac{m^{2}}{n^{2}} L_{0} \gamma^{2}-E^{\prime}\right) .
$$

By comparing (4) with (20) we obtain that the kinetic terms coincide for

$$
u=y, \quad \gamma=-y, \quad q=\frac{m}{n} x+x_{0} .
$$

The choice of $x_{0}=-\frac{a_{2}}{a_{1}}$ allows to write $a_{1} q+a_{2}=a_{1} \frac{m}{n} x$ and, consequently, we have the identifications

$$
\frac{m^{2}}{n^{2}}=4 k^{2}, \quad L_{0}=\frac{\omega^{2}}{4 k^{2}}, \quad c_{1}=a_{1}^{2} b, \quad E^{\prime}=E+4 c_{2} k^{2} .
$$

Therefore, the first integral is

$$
K=\tilde{W}^{\frac{\mu}{2}} G_{\nu},
$$

where

$$
\begin{aligned}
& \tilde{W}=-\frac{2 \mu}{\nu^{2}} y p_{y} X_{L}-8 k^{2}-4 \omega^{2} y^{2}+2 E+8 c_{2} k^{2}, \\
& L=\frac{1}{8 k^{2}} p_{x}^{2}+\frac{\omega^{2}}{4} x^{2}+\frac{b}{4 k^{2} x^{2}}+c_{2},
\end{aligned}
$$

with $(\mu, \nu)=(m, n)$ for $m$ even, $(\mu, \nu)=(2 m, 2 n)$ for $m$ odd, and $G_{\nu}$ given by the recursion [7]

$$
G_{1}=a_{1} x p_{x}, \quad G_{n+1}=X_{L}\left(G_{1}\right) G_{n}+\frac{1}{n} G_{1} X_{L}\left(G_{n}\right) .
$$

We remark that other choices of rescaling and changes of parameters are possible, leading to different (but essentially equivalent) $L$ and $\tilde{W}$.

\section{Conclusions}

In this article we proved that, for any modified extension, there exists a specific CCM of it which maintains the most distinctive property of an extension: the determination of a first integral via powers of an operator applied to a suitable function. This fact suggests the definition of 
a new class of Hamiltonian systems including the Post-Winternitz system as well as all extended Hamiltonian systems.

In [8] we introduced the idea of warped product of Hamiltonian systems. Given two symplectic, or Poisson, manifolds $M$ and $N$ with Hamiltonians $H_{M}$ and $H_{N}$, we consider on $M \times N$, endowed with the product of the symplectic or Poisson structures of $M$ and $N$, the Hamiltonian

$$
H=\alpha H_{M}+\beta H_{N}
$$

where $\alpha$ and $\beta$ are functions on $M \times N$ and we call it the "warped product" of $H_{M}$ and $H_{N}$. If $H_{M}, H_{N}$ are natural Hamiltonians, $M, N$ are cotangent bundles with Riemannian manifolds $B_{M}, B_{N}$ as base manifolds and $\alpha, \beta$ are functions of the product $B_{M} \times B_{N}$, then the metric tensor of $H$ is the standard warped product of the metrics on $B_{M}, B_{N}$.

All extended Hamiltonian systems, together with their CCM considered in this paper, are clearly the warped product of two Hamiltonians: one depending on $\left(u, p_{u}\right)$ solely, the other being $L$. Indeed, the symplectic structure we are using on $H$ is simply the product of the lowerdimensional canonical symplectic structures. Therefore, we may imagine a class of Hamiltonian systems of "warped-power" type determined as follows

- their Hamiltonian $H$ is the warped product of a finite number of other Hamiltonians,

- $H$ admits a constant of the motion determined by the power of an operator applied to some suitable function defined on the same domain of $H$.

Such a class includes all the systems we call "extensions of Hamiltonian systems", together with their CCM as described in this paper, and is naturally parametrized by a natural number at least: the power of the operator generating the first integral.

Finally, Remark 2 suggests a new direction of research, by proposing an alternative form of the operator involved in the extension procedure.

\section{References}

[1] Borisov A.V., Kilin A.A., Mamaev I.S., Superintegrable system on a sphere with the integral of higher degree, Regul. Chaotic Dyn. 14 (2009), 615-620.

[2] Boyer C.P., Kalnins E.G., Miller Jr. W., Stäckel-equivalent integrable Hamiltonian systems, SIAM J. Math. Anal. 17 (1986), 778-797.

[3] Chanu C., Degiovanni L., Rastelli G., Superintegrable three-body systems on the line, J. Math. Phys. 49 (2008), 112901, 10 pages, arXiv:0802.1353.

[4] Chanu C.M., Degiovanni L., Rastelli G., Generalizations of a method for constructing first integrals of a class of natural Hamiltonians and some remarks about quantization, J. Phys. Conf. Ser. 343 (2012), 012101, 15 pages, arXiv:1111.0030.

[5] Chanu C.M., Degiovanni L., Rastelli G., Superintegrable extensions of superintegrable systems, SIGMA 8 (2012), 070, 12 pages, arXiv:1210.3126.

[6] Chanu C.M., Degiovanni L., Rastelli G., Extensions of Hamiltonian systems dependent on a rational parameter, J. Math. Phys. 55 (2014), 122703, 11 pages, arXiv:1310.5690.

[7] Chanu C.M., Degiovanni L., Rastelli G., The Tremblay-Turbiner-Winternitz system as extended Hamiltonian, J. Math. Phys. 55 (2014), 122701, 8 pages, arXiv:1404.4825.

[8] Chanu C.M., Degiovanni L., Rastelli G., Warped product of Hamiltonians and extensions of Hamiltonian systems, J. Phys. Conf. Ser. 597 (2015), 012024, 10 pages.

[9] Hietarinta J., Grammaticos B., Dorizzi B., Ramani A., Coupling-constant metamorphosis and duality between integrable Hamiltonian systems, Phys. Rev. Lett. 53 (1984), 1707-1710.

[10] Kalnins E.G., Kress J.M., Miller Jr. W., Tools for verifying classical and quantum superintegrability, SIGMA 6 (2010), 066, 23 pages, arXiv:1006.0864. 
[11] Kalnins E.G., Kress J.M., Miller Jr. W., Superintegrability in a non-conformally-flat space, J. Phys. A: Math. Theor. 46 (2013), 022002, 12 pages, arXiv:1211.1452.

[12] Kalnins E.G., Miller Jr. W., Post S., Coupling constant metamorphosis and Nth-order symmetries in classical and quantum mechanics, J. Phys. A: Math. Theor. 43 (2010), 035202, 20 pages, arXiv:0908.4393.

[13] Maciejewski A.J., Przybylska M., Yoshida H., Necessary conditions for classical super-integrability of a certain family of potentials in constant curvature spaces, J. Phys. A: Math. Theor. 43 (2010), 382001, 15 pages, arXiv:1004.3854.

[14] Miller Jr. W., Post S., Winternitz P., Classical and quantum superintegrability with applications, J. Phys. A: Math. Theor. 46 (2013), 423001, 97 pages, arXiv:1309.2694.

[15] Post S., Winternitz P., An infinite family of superintegrable deformations of the Coulomb potential, J. Phys. A: Math. Theor. 43 (2010), 222001, 11 pages, arXiv:1003.5230.

[16] Rajaratnam K., McLenaghan R.G., Killing tensors, warped products and the orthogonal separation of the Hamilton-Jacobi equation, J. Math. Phys. 55 (2014), 013505, 27 pages, arXiv:1404.3161.

[17] Rañada M.F., Master symmetries, non-Hamiltonian symmetries and superintegrability of the generalized Smorodinsky-Winternitz system, J. Phys. A: Math. Theor. 45 (2012), 145204, 13 pages.

[18] Rañada M.F., The Tremblay-Turbiner-Winternitz system on spherical and hyperbolic spaces: superintegrability, curvature-dependent formalism and complex factorization, J. Phys. A: Math. Theor. 47 (2014), 165203, 9 pages, arXiv:1403.6266.

[19] Rañada M.F., Santander M., Superintegrable systems on the two-dimensional sphere $S^{2}$ and the hyperbolic plane $H^{2}$, J. Math. Phys. 40 (1999), 5026-5057.

[20] Tashiro Y., Complete Riemannian manifolds and some vector fields, Trans. Amer. Math. Soc. 117 (1965), $251-275$.

[21] Tremblay F., Turbiner A.V., Winternitz P., An infinite family of solvable and integrable quantum systems on a plane, J. Phys. A: Math. Theor. 42 (2009), 242001, 10 pages, arXiv:0904.0738.

[22] Verrier P.E., Evans N.W., A new superintegrable Hamiltonian, J. Math. Phys. 49 (2008), 022902, 8 pages, arXiv:0712.3677. 